\title{
Compléments et corrections à la faune des Ephéméroptères d'Afrique du Nord \\ 2. Le genre Choroterpes Eaton, sous-genre Euthraulus Barnard (Ephemeroptera)
}

\author{
B. Vittel \\ A.G.B. Thomas 2
}

Mots clés : Ephemeroptera, Choroterpes (Euthratlus), Afrique du Nord, élevage de larves, n. comb., n. syn.

L'élevage de larves jusqu'à l'imago (o) a montré que la combinaison Choroterpes (Ch.) lindrothi Peters, 1980 doit être remplacee par Ch. (Euthraulus) lindrothi Peters, 1980, n. comb. et que Ch. (E.) mauritanicus Soldan et Thomas, 1983 en est synonyme (n. syn.).

Additions and corrections to the Ephemeroptera fauna of North Africa

2. The genus Choroterpe's Eaton, subgenus Eathrailus Barnard.

Keywords : Ephemeroptera, Choroterpes (Euthraults), North-Africa, rearing of larvae, n. comb., n. syn.

The rearing of larvae to the male imago stage has shown that the combination Choroterpes (Ch.) lindrothi Peters, 1980 must be replaced by Ch. (Euthraulus) lindrothi Peters 1980, (n. comb.) and that Ch. (E.) mauritanicus Soldan \& Thomas 1983 is a synonym (n. syn.).

\section{Le sous-genre Euthraulus Barnard}

Dans les pays circum-méditerranéens, le sousgenre Euthraulus Barnard, 1932 est l'un des plus mal connus - aucune imago $\sigma$ n'en a encore été décrite - malgré trois espèces signalées :

- Choroterpes (Euthraulus) balcanicus (Ikonomov, 1961) de Macédoine ;

- Ch. (E.) mauritanicus Soldan et Thomas, 1983 d'Algérie ;

- Ch. (E.) assimilis Gaino et Sowa, 1985 de l'île grecque de Lesbos.

1. Laboratoire de Faunistique, Département de Biologie, Faculté des Sciences, Universite S Med Ben Abdallah, B.P. 1796 Atlas, Fès, Maroc.

2. Laboratoire d'Hydrobiologie, UA 695 du C.N.R.S., Université Paul Sabatier, 118, route de Narbonne, 31062 Toulouse Cédex. France.
La récolte d'imagos de ce sous-genre étant rare, les descriptions ont été effectuées jusqu'ici exclusivement (matritanicus) ou essentiellement (balcanicus et assimilis) sur du materiel larvaire.

L'élevage jusqu'à l'imago de larves âgées de $C h$. (E.) mauritanicus, provenant du Rif (Maroc), a montré qu'il s'agissait d'une espèce décrite antérieurement sous une autre combinaison, incorrecte : Cho roterpes $(\mathrm{C} h$.) lindrothi Peters, 1980. Le matériel typique se composait seulement d'une imago $\sigma$.

D'ailleurs les critères de séparation des deux sousgenres Euthraulus et Choroterpes s.s., à l'état imaginal, proposés par Peters et Edmunds dans leurs importants travaux sur la région éthiopienne (1964) et I'hémisphère Est (1970) se révèlent assez incertains pour la région méditerranéenne où ils devront être redéfinis. 
En particulier, sur l'aile postérieure, l'asymétrie du contour des régions proximale el distale, de part et d'autre de la saillie costale - notable sur des espèces d'Euthraulus éthiopiennes (voir par exemple Gillies 1957. Pe.ers et Edmunds 1964) - est pratiquement inexistante sur les espèces circumméditerranéennes (voir Ikonomov 1961, Gaino et Sowa 1985, présent travail fig. 2). Il paraît en être de même pour les deux especes récemment décrites d'U.R.S.S. par Kluge (1984).

Par contre, la séparation des deux sous-genres se fait trìs aisćment à l'é tat larvaire, sur la structure des branchies 2 à 7 , sans tendance franchement internédiaire à notre connaissance. La presence de trois prolongements distaux étroits el subégaux par feuillet (fig. 1) nous permet de ranger sans ambiguité l'espece lindrothi dans le sous-genre Euthraulus et non Choroterpes s.s. Nous proposons donc la combinaison nouvelle:

Choroterpes (Ch.) lindrothi Peters, 1980

$=$ Choroterpes (Euthraulus) lindrothi Peters, 1980. 11. comb.

Cetle demiere entraîne malheureusement la chute en synonymic de la désignation $C h$. (E.) mauriani'tus Soldan et Thomas, 1983, érigée à l'époque parce que censée s'appliquer à la première espèce endemique - nord-africaine connue du sous-genre Euthraulus.

En effet, l'imago or et ses genitalia correspondent bien à la description de lindrothi par Peters.

Nous proposons donc aussi la synonymie:

Choroterpes (Euthraulus) mauritanicus Soldan et Thomas, 1983

= Choroterpes (Euthraulus) lindrothi Peters, 1980. n. syn.

\section{Choroterpes (Euthraulus) lindrothi Peters, 1980}

Imago or

Tête.

Face et vertex brun foncé, y compris la forte carène interoculaire postérieure à l'ocelle median.

Scape et pédicelle brun moyen, funicule brun grisâtre.

Yeux nettement séparés ; partie supérieure brun rouge, plus claire ventralement; partie inférieure noirâtre.
Thorax.

Prothorax brun soutenu dorsalement et ventralement.

Mesothorax : scutum brun foncé brillant ; scutellum noirâtre latéralement : pleurites et sternite bnun brillant.

Metathorax brun moyen dorsalement, plus clair ventralement.

Pattes brun moyen terne assez uni, sauf aux abords de l'articulation fémur-tibia I parfois plus foncés. Tarses II et III grisâtres.

Ailes antérieures à membrane hyaline, sauf dans la région proximale, bistre jusqu'au pont costal. Nervation longitudinale brun grisâtre peu marquée sauf $\mathrm{Sc}$ et R1. Nervation transversale très claire et peu visible. Longueur de la tige de la médiane (MA1 + MA2) supérieure à la longueur de MA2 sur les spécimens mesurés (voir aussi Peters 1980, fig. 1). Aire ptérostigmatique légèrement laiteuse avec de 10 à 15 nervures transverses peu ou pas ramifiées.

Ailes postérieures hyalines; saillie costale non matérialisée par un décrochement distal (fig. 2 ; voir aussi Peters t. c., Fig. 2-3). Nervures transverses plus foncées qu'aux ailes antérieures.

Abdomen.

Tergites brun terne assez uniforme - plus ou moins translucides selon les individus - à l'exception d'une étroite ligne claire médio-dorsale surtout visible à partir du $4^{e}$ segment. Ce caractère distingue net tement Ch. (E.) lindrothi des deux Choroter. pes (s.s.) nord-africains. Bords des tergites plus foncés contre les pleures.

Sternites 1 à 8 plus clairs que les tergites, olivâtres. Stemite 9 blanchâtre, sauf une maculation bordante, brun intense, en croissant à concavité postérieure.

Cerques brun grisâtre clair à annélation très discrète près des articulations.

Genitalia.

Styligère dépourvu d'encoche médiane (fig. 3). Article basal des styles épais, concave seulement sur la moitié distale : saillie interne assez anguleuse. saillie basale externe peu marquée.

Lobes péniens arrondis à l'apex en vue ventrale (fig. 3 et 5, voir aussi Peters t.c., fig. 4), plus ou moins divergents selon les individus, présentant en fait un rétrécissement subapical ventral netternent visible de profil (fig. 6 et 7). L'apex des lobes porte quelques épines. 


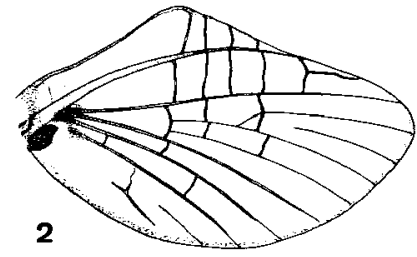

0.5
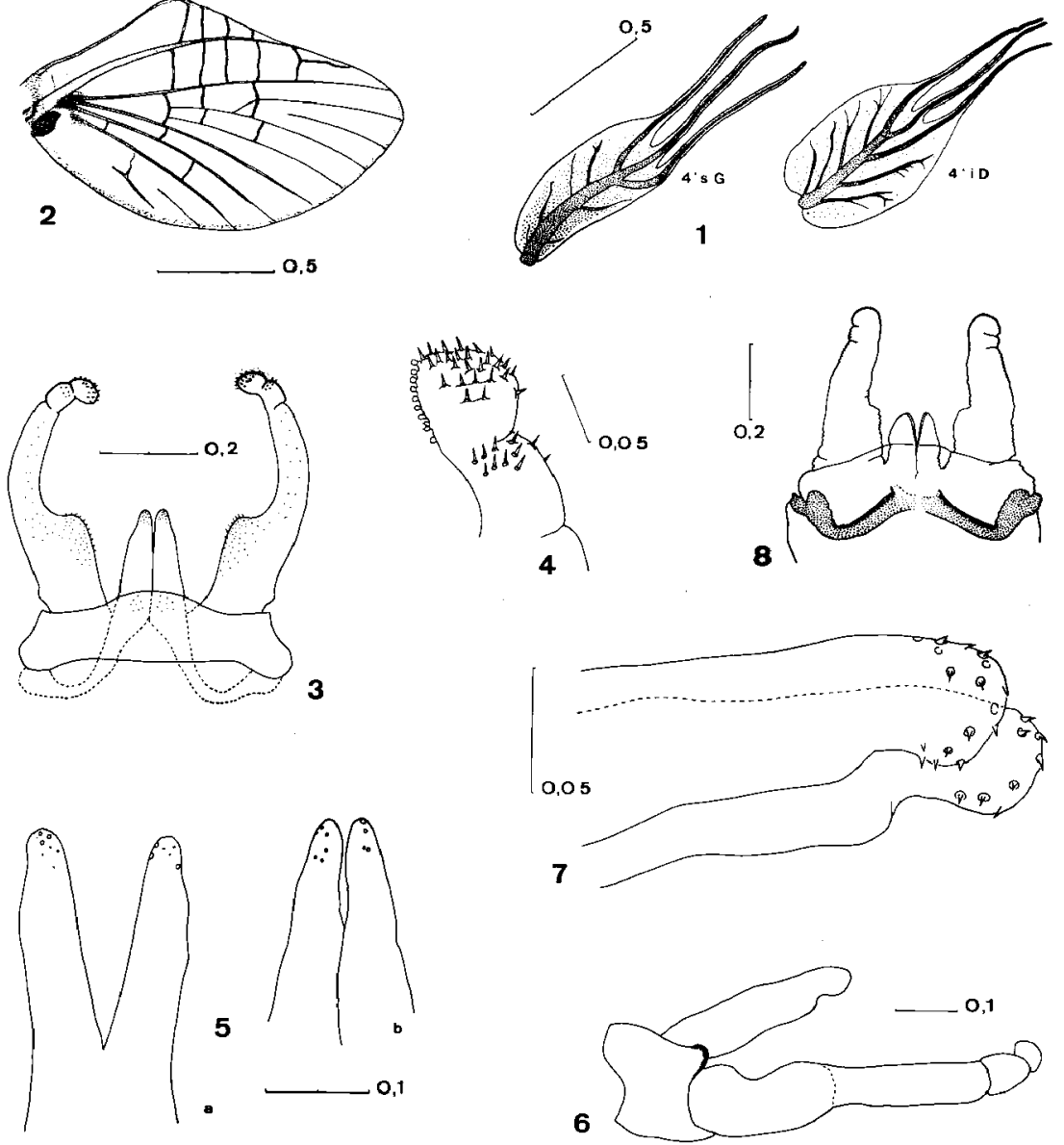

Fig. 1 à 8 : Choroterpes (Euthraulus) lindrothi Peters. Echelle en mm.

Fig. 1 : feuillets branchiaux de la $4^{\mathbf{e}}$ paire (sG : supérieur gauche ; iD : inférieur droit) sur une larve mature élevée jusqu'à l'imago $\left(O^{*}\right)$.

Fig. 2 à 7 : imago $\% .2$ : aile postérieure. 3 : genitalia en vue ventrale. $4: 2^{\epsilon}$ et $3^{c}$ articles des st yles. 5 a et $b:$ lobes péniens de deux individus en vue ventrale. $6:$ genitalia en vue latérale. $7:$ détail de la moitié distale des lobes péniens en vue latérale.

Fig. 8 : genitalia de la subimago or en vue ventrale. 


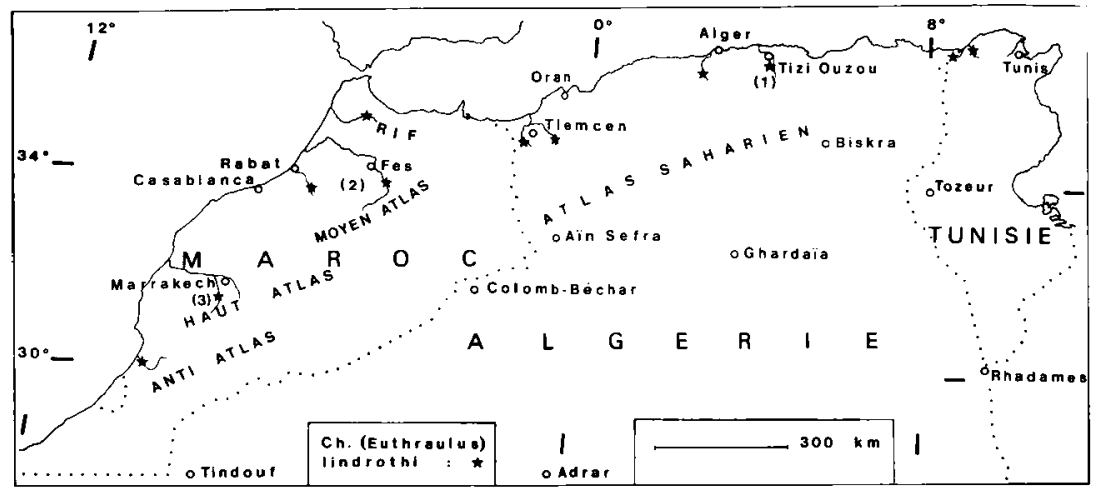

Fjg. 9 ; répartition de Choroterpes (Euhlraulus) lindrothi Peters en Afrique du Nord (rélérences citées d'Esl en Ouest). Materiel larvaire seulement, sauf la citation du Rif (élevage); une seule mention par cours d'eau principal.

Tunisie : Boumaiza et Thomas (1986). Algérie : $(1)=0$. Aïssi (A. Thomas dét, in Lounaci 1987); Soldan et Thomas (1983); Gagneur et Thomas (1988). Maroc : Rif = présentes récoltes; Moyen Aulas (2) = O. Sebou (Dakki 1979, s.n. Aprionyx ? sp.) et O. Bou Regreg (Dakki et El Agbani 1983, s.n. Choroterpes (Euthraulus) sp.); Haut Atlas (3) = O. N'Fiss (A. Thomas dét., A. Ajakane leg.): Anti Atlas = O. Massa (Peters 1980).

Taille.

Longueur de l'aile antérieure : 6 à $6,5 \mathrm{~mm}$.

\section{Subimago o}

Couleurs plus ternes et en général plus claires que chez l'imago.

Mesonotum, en particulier, brun orangé moyen. Ailes gris uniforme.

Les tergites abdominaux, foncés, portent des taches claires : l'une, linéaire et médiane, très nette, l'autre plus large et submarginale, près des pleures.

Sternites blanc jaunâtre.

Genitalia : fig. 8.

\section{Matériel examiné et répartition}

33 imagos et subimagos (B.V. leg.) conservées en alcool dans les collections des auteurs (dont $3 \mathrm{i}$. $\sigma$ et $1 \mathrm{~s}$. $\sigma$ à Toulouse).

Provenance : massif du Rif (Maroc septentrional) ; l'oued Loukos à $150 \mathrm{~m}$ (pont sur la route $P 28$ entre Ouezzane et Chechaouen).
Captures : 5 i. $\sigma$ (10.V-1987). Elevages, à partir de cette date ; 8 i, $\sigma$ et $2 \mathrm{~s}, \sigma, 7 \mathrm{i}$. $Q$ et $11 \mathrm{~s}$. $\%$. La période de vol débute en fin mars à cette station et, nettement plus au Sud, la capture du type a été effectuée un 2 mars.

D'autre part, l'un de nous (A.T.) a examiné plus de 500 larves provenant de toute l'Afrique du Nord, régions désertiques exceptées (fig. 9). Probablement endémique du Maghreb, $C h$. (E.) lindrothi est assez répandue mais en général moins abondante et moins fréquente que l'espèce de Leptophlebiidae la plus commune en Afrique du Nord: $C h$. (Ch.) atlas (voir Thomas \& Vitte 1988).

Travaux cités

Boumaiza (M.) \& Thomas (A.G.B.). 1986. - Répartition et écolo gie des Ephéméroptères de Tunisie ("*r partie) (Insecta, Ephemeroptera). Archs Inst. Pasteur Tumis, 63 (4) : 567.599.

Dakki (M.). 1979. - Recherches hydrobiologiques sur un cours deau du Moyen Atlas (Maroc). Thèse de spécialité, Unit. Aix. Marseille (St Jéróme), $126 \mathrm{p}$. + VIII.

Dakki (M.) \& El Agbani (M.A.). 1983. - Ephémèroptères d'Afrique du Nord. 3, éléments pour la connaissance de la faune marocaine. Bull. Inst. scientl., Rabat, $7: 115-126$. 
Gagneur (J.) \& Thomas (A.G.B.). 1988. - Contribution à la connaissance des Ephéméroptères d'Algérie. I. Répartition et écologie (1ere partie) (Insecta, Ephemeroptera), Bull. Soc. Hist. nat. Toulouse, $124: 213-223$.

Gaino (E.) \& Sowa (R.). 1985. - Nouvelles especes de Leptophlebiidac de Grèce et de Yougoslavie (Ephemeroptera). Boll. Soc. ent. ilal., $117(1-3)$ : 8-17.

Gillies (M.T.). 1957. - New records and species of Euhrautis Barnard (Ephemeroptera) from East Africa and the oriental region. Proc. $R$. ent. Soc: London, $26: 43-48$.

Ikonomov (P.). 1961. - Euthraulus balcanicus sp. n. (Leptophlebiidae). Fragm. halcan., 4 (1): 1-10.

Kluge (N. Yu.). 1984 . Mayflies of the subgenus Euthraulus Barn. (Ephemeroptera, Leptophlebiidae, genus Choroterpes) of the fauna of the USSR. Ent. Obozr. 63 (4) : 722-728.

Lounaci (A.). 1987. - Recherches hydrobiologiques sur les peuplements d'invertébrés benthiques du bassin de l'oued Aïssi (Grande Kabylie). Thèse de Magister, Univ. Alger, 133 p. + VII.
Peters (W.L.). 1980. - Choroterpes (Chororerpes) lindrothi, a new species of mayfly from Morocco (Epheme roptera : Leptophlebiidae). Entomologia Generalis, 6 (2-4) : 371-373.

Peters (W.L.) et Edmunds (G.F.). 1964. - A revision of the generic classification of the Ethiopian Leptophlebiidae (Ephemeroptera). Trans. $R$. ent. Soc. London, 116 (10): 225-253.

Peters (W.L.) et Edmunds (C.F.). 1970. - Revision of the generic classification of the eastern hernisphere Le ptophlebiidae (Ephemeroptera). Pacif. Insects, 12 (1): 157-240.

Soldan (T.) et Thomas (A.G.B.). 1983. - New and little-known species of mayflies (Ephemeroptera) from Algeria. Acta ent. bohemoslov, $80: 356.376$

Thomas (A.G.B.) et Vitle (B.). 1988. - Complèments et corrections à la faune des Ephemeropteres d'Afrique du Nord. 1. Le genre Choroterpes Eaton sensu stricto (Ephemeroptera). Annls Limnol. 24 (1) : $61-65$. 